\title{
1 Seasonal forecasting of groundwater levels in principal aquifers of the United
}

\section{Kingdom}

3

4 J.D. Mackay ${ }^{a *}$, C.R. Jackson ${ }^{a}$, A. Brookshaw ${ }^{b}$, A.A. Scaife ${ }^{b}, J^{\prime}$ Cook $^{c}$ and R.S. Ward ${ }^{a}$. 5

$6{ }^{a}$ British Geological Survey, Environmental Science Centre, Keyworth, Nottingham NG12 $7 \quad 5 G G, U K$

$8{ }^{\mathrm{b}}$ Met Office, FitzRoy Road, Exeter, EX1 3PB, UK

$9 \quad{ }^{\mathrm{C}}$ ISA Lille, Lille, France.

10 * Corresponding author: Tel.: +44 1159 363100; fax: +44 1159 363200; email:

11 joncka@bgs.ac.uk

\section{Abstract}

14 To date, the majority of hydrological forecasting studies have focussed on using medium-

15 range (3 to 15 days) weather forecasts to drive hydrological models and make predictions of

16 future river flows. With recent developments in seasonal (1 to 3 months) weather forecast

17 skill, such as those from the latest version of the UK Met Office global seasonal forecast system (GloSea5), there is now an opportunity to use similar methodologies to forecast

19 groundwater levels in more slowly responding aquifers on seasonal timescales. This study

20 uses seasonal rainfall forecasts and a lumped groundwater model to simulate groundwater

21 levels at 21 locations in the United Kingdom up to three months into the future. The results

22 indicate that the forecasts have skill; outperforming a persistence forecast and demonstrating reliability, resolution and discrimination. However, there is currently little to 
24 gain from using seasonal rainfall forecasts over using site climatology for this type of

25 application. Furthermore, the forecasts are not able to capture extreme groundwater levels,

26 primarily because of inadequacies in the driving rainfall forecasts. The findings also show

27 that the origin of forecast skill, be it from the meteorological input, groundwater model or

28 initial condition, is site specific and related to the groundwater response characteristics to

29 rainfall and antecedent hydro-meteorological conditions.

31 Keywords

32 Seasonal forecasting; ensemble forecasting; groundwater level forecasting; AquiMod;

33 GloSea5.

34

35

36

37

38

39

40

41

42

43

44 
1. Introduction

46 Often a cleaner and more reliable source of drinking water than surface reservoirs,

47 groundwater aquifers comprise the world's largest freshwater resource and provide

48 resilience to climate extremes which may increase in frequency with future climate change

49 (Alley et al., 2002; Mishra and Singh, 2010; Sukhija, 2008). Under prolonged dry climatic

50 conditions groundwater drought can develop, often characterised by significantly low

51 groundwater levels which persist for months to years (Lanen and Peters, 2000; Marsh et al.,

52 2007). This may lead to the drying up of significant water-bearing wells and the degradation

53 of ecologically important rivers and springs. Conversely, lasting wet conditions can induce

54 anomalously high groundwater levels resulting in persistent flooding, potentially at large

55 economic cost (Huntingford et al., 2014; Pinault et al., 2005; Upton and Jackson, 2011).

Proper management of these resources is vital to ensure their sustainability and to reduce

57 the risk and impacts from groundwater level extremes.

58

59 One possible way forward is to forecast future groundwater levels so that management

60 strategies can be employed in advance of likely future events. However, these approaches

61 generally require some insight into future weather patterns and an understanding of site-

62 specific hydrogeological characteristics that control the non-linear groundwater discharge

63 response to changes in groundwater levels (Eltahir and Yeh, 1999; Moore and Bell, 1999).

64 This paper attempts to do this by using state-of-the-art seasonal weather forecasts to drive

65 a series of groundwater models to forecast groundwater levels up to three months into the 66 future.

67 
68

69

70

71

72

73

74

75

76

77

78

79

80

81

82

83

84

85

86

87

88

89

90

The majority of groundwater level forecasting studies have been conducted using black-box modelling approaches (Jakeman et al., 2006) whereby an empirical relationship between groundwater level time-series and one or more predictor variables is found using an optimization algorithm (Sahu, 2003). Typically, meteorological covariates, including rainfall and temperature, are used because these perturb groundwater recharge fluxes. Flow through the unsaturated zone and saturated aquifer can slow the response of groundwater level to rainfall events (Alley et al., 2002). Accordingly, a suitable characterisation of this lagged response may be sufficient for forecasting future groundwater levels in aquifers, given up-to-date weather data.

The most widely used method to characterise the lagged response of groundwater levels to meteorological predictor variables is the non-parametric Artificial Neural Network (ANN), a flexible tool that is able to implement multiple statistical models to replicate patterns in time-series (Maier and Dandy, 2000). Daliakopoulos et al. (2005) used neural networks to forecast monthly groundwater levels in a highly heterogeneous alluvial aquifer in Crete, Greece. Trichakis et al. (2009) also used ANNs to forecast the change in hydraulic head in a complex karstic limestone aquifer in Greece which proved to be accurate up to a 90-day lead time. Taormina et al. (2012) forecast groundwater levels on an hourly time-step for a flashy shallow coastal aquifer in the Venice lagoon and found that they could accurately reproduce groundwater depths for several months ahead. These, along with other studies that have used ANNs (Nourani et al., 2008; Sreekanth et al., 2009; Ying et al., 2014) all show significant forecasting skill months into the future. However, there are two key limitations with these approaches: i) not all aquifers exhibit a significant lagged response to antecedent 
91 weather; and ii) to forecast more than one time-step ahead these studies used retrospective

92 observed meteorological predictor variables which would not be available ahead of time.

93

94 Tsanis et al. (2008) recognised the second issue and adapted the work of Daliakopoulos et al. (2005) to include a precipitation projection model which, if used in combination with seasonally averaged temperature data, could simulate groundwater levels up to 30 months ahead, achieving a $R^{2}>0.9$. It should be noted, however, that it is likely that this high correlation score largely reflects the model's ability to capture a downward groundwater level trend induced by steady abstractions in the dry season. Even so, it does demonstrate the possibility of using meteorological forecasts to extend the lead time of real-time groundwater level projections.

Alternative black box methods such as support vector machines (Behzad et al., 2010;

Suryanarayana et al., 2014; Vapnik, 1999; Yoon et al., 2011) and wavelet decompositions (Adamowski and Chan, 2011; Maheswaran and Khosa, 2013; Partal and Kişi, 2007) have also been used for groundwater level forecasting in the past with promising levels of skill. Mendicino et al. (2008) took a different approach by using a simple conceptual distributed water balance model to derive average groundwater storage over the most southern peninsular of Italy, the outputs of which were used to derive a groundwater drought index.

110 They found that due to the persistence of low groundwater levels in the summer months, 111 droughts could be forecast months prior to their occurrence based on model simulations of 112 the current groundwater storage. 
114 While these studies have shown some skill, the relative infancy of groundwater level

115 forecasting science becomes apparent when compared to the abundance of studies

116 focussed on forecasting other hydrological variables such as river discharge for flood

117 forecasting (see Cloke and Pappenberger (2009) and Cuo et al. (2011) for two

118 comprehensive reviews of these applications). Here, forecasters are not afforded the luxury

119 of long response times to prior weather patterns. At the catchment scale, river flow

120 response time to rainfall is typically of the order of minutes to hours. As such, forecasters

121 drive their hydrological models with medium-range weather forecast products from

122 numerical weather prediction (NWP) centres, which typically offer lead times of 3 to 15

123 days. These extended lead times may allow water resource managers and contingency

124 planners to implement mitigation strategies in advance of extreme events. Of course, the

125 benefit of increased lead time comes at a cost; namely that these meteorological products

126 are inherently uncertain due to the non-linear, chaotic nature of the atmosphere (Lorenz,

127 1963). In response, river flow forecasters now adopt probabilistic methodologies that

128 incorporate this uncertainty rather than relying on a single deterministic forecast. A popular

129 approach that couples probability with determinism is ensemble forecasting (Lewis, 2005)

130 whereby a number of deterministic weather forecasts with differing initial conditions are

131 used to drive the hydrological model. If these realisations are assumed independent and of

132 the same random process, it is possible to assign probabilities to the occurrence or

133 exceedance of given flow thresholds. This probabilistic, ensemble-based approach provides

134 more consistent and skilful outlooks from which users can manage risks more effectively

135 (Addor et al., 2011; Buizza, 2008). One may also cascade other uncertainties, such as those

136 associated with the hydrological model parameterisation, through the forecasting system

137 (Beven, 2006; Pappenberger et al., 2005; Zappa et al., 2010; Zappa et al., 2011). A well 
138

139

140

141

142

144 The response of groundwater levels to rainfall generally operate on longer time scales (days

established approach for this is the Generalised Likelihood Uncertainty Estimation (GLUE)

method (Beven and Binley, 1992; Beven and Binley, 2013), whereby an informal likelihood

function is used to weight an ensemble of behavioural models. It should be noted, however,

that due to the computational burden, such approaches for real-time hydrological

forecasting applications are still not widely used today.

145 to months) than river flows. As such, strategies to mitigate an imposing groundwater

146 drought, for example, can only be properly formulated with a good understanding of the

147 likely future groundwater levels over a similar time scale. Here, longer-range weather

148 forecasts on the scale of months would be required, like those produced by the latest

149 version of the Met Office global seasonal forecast system (GloSea5) which are now showing

150 increased skill up to a three month lead time (Scaife et al., 2014). To date, however, the

151 majority of seasonal forecasting studies have been undertaken by the river flow forecasting

152 community. Yossef et al. (2012) investigated the potential for forecasting monthly and seasonal river flow extremes in 20 large river basins around the world by driving the global

hydrological model, PCR-GLOBWB (Sperna Weiland et al., 2010) with observed

155 meteorological forcing data. They found that they could capture observed flood and

156 drought events given skilful meteorological inputs. More recently, Svensson et al. (2015)

157 used GloSea5 seasonal rainfall forecasts to drive a $1 \mathrm{~km}$ resolution water balance model

158 (Bell et al., 2013) and forecast winter (December-January-February) river flows across the

159 UK. The forecasts correlated with observed winter river flows with a median correlation

160 score of 0.45 . They also found a clear geographical contrast in the source of predictability

161 whereby the initial condition was the strongest source of predictability in the more 
162 permeable, baseflow-dominated catchments of south-east England, and the skill was much

163 more dependent on the meteorological forcing data for the flashy catchments in the north-

164 west of Great Britain. The role of river flow response characteristics on seasonal forecast

165 skill was also found to be important for global seasonal river flow forecasting by Yossef et al.

166 (2013). Indeed, contrasting response characteristics to rainfall can also be found in

167 groundwater level time-series (e.g. see the work of Bloomfield and Marchant, 2013), and

168 these are likely to influence the sensitivity of groundwater level forecasts to the

169 meteorological forcing data.

170

171 To summarise, skilful forecasts of groundwater levels would provide useful information to

172 water resource managers and contingency planners which could help to mitigate hazards

173 such as groundwater flooding and drought, both of which can lead to social, economic and

174 environmental degradation. Experience gained from the river flow forecasting community

175 shows that skilful ensemble hydrological forecasts can be generated using driving data from

176 medium-range NWP models. However, because aquifers generally respond to prevailing

177 weather patterns over a number of months, the insight gained over a 15-day lead time may

178 be small. This has led most studies to rely on the lagged response of groundwater levels to

179 past weather patterns to make forecasts. However, it may be possible to extend the skilful

180 forecast lead time using seasonal weather forecast products to drive groundwater models,

181 an approach that is already showing some skill in river flow forecasting experiments.

182

183 This paper presents a new probabilistic groundwater level forecasting approach that utilises

184 state-of-the-art GloSea5 multi-member seasonal forecasts of rainfall produced by the UK

185 Met Office to drive a series of groundwater models up to three months into the future. A 
186 parsimonious lumped conceptual groundwater model, AquiMod (Mackay et al., 2014),

187 which simulates groundwater levels at observation boreholes has been used. The models

188 have been calibrated to simulate groundwater level time-series at 21 locations across the

189 UK and in different aquifers with contrasting hydrogeological properties and response

190 characteristics to rainfall. The skill of the groundwater level forecasts is evaluated over the

191 four UK seasons using a 14-year sequence of GloSea5 rainfall reforecasts. For comparison,

192 reforecasts using rainfall climatology and observed rainfall have also been evaluated.

193 Consideration of the catchment response characteristics and their influence on forecast skill

194 are also made. From these analyses, this study seeks to provide a first evaluation of the

195 potential for national, real-time seasonal groundwater level forecasting.

\section{Methodology}

\subsection{Study catchments}

198 In total, 21 groundwater catchments, each with an observation borehole and associated 199 groundwater level record were selected for this study from a database of 181 groundwater 200 level time-series held in the National Groundwater Level Archive (Marsh and Hannaford,

201 2008). They were selected because: i) they are situated in unconfined aquifers for which the

202 AquiMod groundwater model is best suited; ii) they are located away from any significant

203 groundwater abstractions; and iii) they have continuous monthly groundwater level records 204 that cover the operational 14-year GloSea5 reforecast period from March 1996 to February 2052010 (MacLachlan et al., 2014) and at least 15 years of data prior to this for model

206 calibration. The boreholes penetrate into some of the UK's principal aquifers including the

207 Cretaceous Chalk and Lower Greensand, the Jurassic and Magnesian Limestone and the 
Permo-Triassic Sandstone (Figure 1). Between 16 and 34 years of continuous groundwater level data were available for model calibration.

Figure 2 shows the raw groundwater level time-series for four of the observation boreholes.

212 Also included are the groundwater level auto-correlation plots and the rainfall-groundwater

213 level cross-correlation plots. It can be seen that groundwater level fluctuations contrast

214 between the catchments. For example, Ashton Farm shows a sinusoidal pattern with

215 relatively uniform amplitude while the New Red Lion borehole shows more variable

216 amplitude with multiple winter peaks. The West Dean cross-correlation plot shows the

217 highest correlation between groundwater and rainfall at a lag of zero, indicating a very rapid

218 and flashy response. This is in contrast to the smooth Heathlanes hydrograph which exhibits

219 relatively small seasonal variability, but more pronounced inter-annual fluctuations. The

220 auto-correlation and cross-correlation plots for this site indicate significant persistence in

221 levels and a much longer response time to rainfall. Also note that because this borehole is in

222 a high storage Sandstone aquifer, annual groundwater levels typically fluctuate by only 0.5

$223 \mathrm{~m}$. In contrast, the water table at New Red Lion in the low porosity Jurassic Limestone

224 aquifer can vary by as much as $20 \mathrm{~m}$ in one year.

\subsection{AquiMod}

226 AquiMod takes monthly rainfall and potential evapotranspiration (PET) driving data and

227 uses conceptual hydrological equations to simulate the downward movement of water

228 through the soil and unsaturated zone and the lateral flow and subsequent discharge of

229 groundwater through the saturated zone (Figure 3). A soil module divides rainfall between

230 evapotranspiration, runoff and soil drainage. The soil drainage is attenuated through the 
231 unsaturated zone using a Weibull distribution transfer function, before reaching the

232 saturated zone as groundwater recharge. Discharge from the saturated zone is calculated

233 using a Darcy flux equation. The reader is referred to Mackay et al. (2014) for a more

234 comprehensive description of the underlying theory and model code.

235

236 The AquiMod code was chosen for this study because it was designed specifically for

237 simulating groundwater levels at observation boreholes. It includes in built Monte Carlo

238 parameter sampling, has a small computational burden and also allows the user to

239 incorporate different saturated zone model structures with variable levels of complexity.

240 Mackay et al. (2014) showed that this model can efficiently capture the non-linear

241 groundwater level dynamics in a range of hydrogeological settings. They also showed that a

242 two or three layer aquifer representation is generally most efficient and these structures

243 have been adopted in this study (Figure 3).

$244 \quad$ 2.3. Model calibration

245 The models were driven with observed monthly rainfall and PET data and calibrated against

246 observed groundwater levels prior to the reforecast period. Rainfall data were obtained

247 from the national $5 \mathrm{~km}$ gridded dataset held by the UK Met Office National Climate

248 Information Centre (Perry et al., 2009). This is comprised of rain gauge data interpolated

249 onto a regular grid using inverse-distance weighting. PET data were extracted from the Met

250 Office Rainfall and Evapotranspiration Calculation System (MORECS) dataset (Field, 1983)

251 which uses synoptic station data in conjunction with a modified version of the Penman-

252 Monteith equation to determine the monthly average PET rate on a $40 \mathrm{~km}$ grid of over the

253 UK (Monteith and Unsworth, 2008). 
255 Following the methodology of Mackay et al. (2014), eight of the possible 16 model

256 parameters were fixed based on known catchment characteristics while the remaining were

257 used as calibration parameters (Table 1). A Monte Carlo procedure was used to randomly

258 select $10^{6}$ unique parameter sets from a user-defined parameter space for each model

259 structure. Here we considered the uncertainty in model structure and parameter selection

260 by adopting the GLUE methodology and using the well established Nash-Sutcliffe efficiency

261 (NSE) score (Bennett et al., 2013; Nash and Sutcliffe, 1970) as the informal likelihood

262 measure. Only those models that exceeded a NSE score of 0.5 were deemed behavioural.

263 Those that did not achieve this were assigned a likelihood of zero.

264

Between 1780 and 2470 behavioural models were obtained for the 21 study catchments

266 achieving a maximum efficiency ( $\mathrm{NSE}_{\max }$ ) between 0.71 and 0.94 and a containment ratio

267 (CR) (Xiong and O'Connor, 2008), which specifies the percentage of observations captured

268 within specified upper and lower prediction bounds, between 65.3 and $97.8 \%$ when using

269 the GLUE 95\% confidence interval (Table 2). Figure 4 shows the observed and simulated

270 groundwater levels with the GLUE 95\% prediction bounds for Bussels, where AquiMod

271 achieved the highest NSE $E_{\max }$, and Therfield Rectory, where AquiMod scored the lowest

$272 N S E_{\text {max }}$. It can be seen that the GLUE prediction bounds for Bussels contain almost all of the

273 observations and the best model closely replicates the timing and seasonality in the

274 hydrograph including the pronounced 1976 drought period. For Therfield Rectory, AquiMod

275 captures the timing and seasonality of the hydrograph and most of the observations during

276 the drought of 1973. However, it fails to capture the rapid recession in 1992, and some of 
277 the peak levels observed in 1961, 1979 and 1988. These deficiencies are considered in more

278 detail in the discussion.

\subsection{Reforecast climate data}

280 Monthly rainfall inputs for the 14-year reforecast period were taken from the GloSea5 model. These comprised four reforecasts per year representing the four seasons: i) spring March-April-May (MAM); ii) summer June-July-August (JJA); iii) autumn September-October-

November (SON); iv) winter December-January-February (DJF). Each consisted of an

winter and summer forecasts were made up of 24 ensemble members while the spring and

autumn forecasts were made up of 12 ensemble members. All were downscaled to the

the relationship between seasonal UK average and seasonal catchment rainfall for the

Ashton Farm and New Red Lion observation boreholes. The fitted linear regression models

the variance. In general, however, the linear regression models demonstrated a good fit,

295 with a mean $R^{2}$ score of 0.46 across the study catchments. These models were then used to downscale the GloSea5 forecasts of UK average rainfall for each catchment. The downscaled

297 GloSea5 seasonal rainfall forecasts for Ashton Farm showed the most skill, where the 
300 Red Lion showed negligible correlation with the observed catchment rainfall (Figure $5 \mathrm{~d}$ ).

301 Overall, the skill of the downscaled rainfall forecasts was low with a mean $R^{2}$ of 0.19 across

302 the study catchments.

303

304 For each seasonal reforecast at a given location, the population of behavioural models were

305 run for two years using observed rainfall and PET data to initialise the soil and unsaturated

306 zone in the models. Their initial groundwater levels were fixed to the latest observation. The

307 models were then run for a further three months using the rainfall and PET data described

308 above, producing an ensemble of predictions with $n * m$ members, where $n$ is the number of

309 behavioural models, and $\mathrm{m}$ is the rainfall ensemble size. The predicted groundwater level

310 probability density function was then constructed using the predefined GLUE likelihoods

311 and assuming equal probability of occurrence for each rainfall ensemble member.

$312 \quad$ 2.5. Skill analysis

313 When evaluating forecast skill, it is often useful to establish categorical events for which the

314 observed and forecast frequencies can be compared. Here, three categorical events were

315 chosen for each catchment; below, near and above normal groundwater levels, defined by

316 monthly terciles from the observed groundwater level data. Jolliffe and Stephenson (2012)

317 detail a vast number of forecast verification metrics. We have chosen to use four

318 quantitative metrics which assess different aspects of forecast skill for a given categorical

319 event including:

320 
321

322

324

327

334 We chose to convert the CRPS into a skill score, the CRPSS, by comparing the groundwater

1. Frequency bias: The ratio of the total number of forecast occurrences to the total number of observed events. Here, the forecast event was defined as that which had the highest forecast probability.

2. Reliability: The consistency between the forecast probabilities and the observed relative frequencies. Here, a negatively oriented reliability score derived from the decomposition of the brier score (Murphy, 1973) has been used.

3. Relative operating characteristic (ROC) score: This measures the capacity to correctly discriminate between the occurrence and non-occurrence of an event. A value greater than 0.5 indicates that the hit rate exceeds the false alarm rate.

4. Continuous ranked probability score (CRPS): Calculated as the integrated square difference between the cumulative distributions of the forecasts and observations. This is a probabilistic generalisation of the mean absolute error. level forecasts to a reference persistence forecast. A persistence-type benchmark was deemed the most rigorous test given that hydrogeological memory can serve as a potential source of skill. We evaluated three different benchmarks against historical observed groundwater levels including i) persisting the latest observed groundwater level; ii) perturbing the latest observed groundwater level using the monthly mean changes in groundwater levels taken from historical data; and iii) persisting the percentile location of the initial groundwater level in the distribution of historical groundwater levels for that month over the following three months (i.e. if the initial condition was near-normal, the forecast for the subsequent three months would remain in this category). We found that 
344 the third approach was the best, consistently outperforming the other two benchmarks and

345 so this was deemed the most rigorous test for forecast skill.

346

347 To complement the benchmark tests, the groundwater models have also been driven with

348 two other meteorological inputs including: i) an unskilful rainfall ensemble made up of re-

349 sampled observed catchment data; and ii) a best case deterministic rainfall input using

350 observed data.

\section{Results}

352 It is known that groundwater levels respond to rainfall differently between the catchments.

353 It is therefore likely that the models will also respond differently. This is examined in the

354 first part of the results by undertaking a sensitivity analysis of the models. The results from

355 this are used to organise the models into a number of response type groups. Note here, and

356 in the text that follows, the term model refers to the population of behavioural models for a

357 given catchment rather than a single model realisation. The remainder of this section

358 analyses the skill of the forecasts for each of the response groups, first by using the skill

359 metrics outlined above and then by analysing a selection of forecast time-series plots.

$360 \quad 3.1$ Groundwater level response to rainfall

361 It is postulated that because of the contrasting response characterises to rainfall across the

362 catchments, the calibrated models will exhibit different sensitivities to rainfall over the

363 three month forecast horizon. Understanding these sensitivities is important because they

364 influence the added value of using seasonal rainfall forecasts to simulate future

365 groundwater levels. 
367 A relative measure of sensitivity to rainfall has been derived for each of the calibrated

368 models. To do this, each model was spun-up using observed rainfall and PET and then run

369 for three months using six arbitrary synthetic rainfall inputs ranging from 0 to $5 \mathrm{~mm} \mathrm{~d}^{-1}$. This

370 process was repeated using each of the months in the reforecast sequence as the initial

371 condition. The sensitivity was then calculated for each month as the range of the six

372 groundwater level forecasts, normalised with respect to the model specific yield. This

373 normalisation step accounts for the different storage properties of each model to allow for

374 easier inter-model comparison.

375

376 Figure 6a shows how the model sensitivity to rainfall changes over the reforecast period for

377 one, two and three month simulations for the Rockley observation borehole in the Chalk

378 aquifer. As would be expected, the sensitivity increases with lead time as the influence of

379 the initial condition diminishes, but there is also a seasonal cycle with peak sensitivity during

380 the winter and considerably reduced sensitivity in the summer months. Given that the

381 climate data for the forecasts are fixed, these variations are a result of perturbations in the

382 initial conditions only. This can be explained by the initial soil moisture deficit (SMD)

383 conditions in the model (Figure 6b) which generally develop in the warmer summer months

384 and must be satisfied before recharge (Figure $6 \mathrm{~d}$ ) is initiated. In the winter months the SMD

385 is small and so small changes in the rainfall input can significantly perturb the modelled

386 groundwater level. Despite this, the sensitivity can increase as the soil moisture deficit

387 develops (for example see year 2003 boxed in Figure 6). This behaviour can be attributed to

388 the initial groundwater level condition, which shows to be receding, and the quadratic

389 groundwater discharge response to a unit rise in groundwater head. In other words, as the 
groundwater level recedes, the discharge response to an influx of recharge is smaller, and so

the sensitivity increases.

392

Similar seasonal fluctuations in sensitivity were observed for all of the study catchments,

but the magnitude varied substantially. The reason for this is likely to be multifaceted, but it

can be attributed primarily to the different model response times to rainfall. It is possible to

investigate this by considering the calibrated unsaturated zone Weibull distribution transfer

function in AquiMod which spreads the flux of water from the soil zone to the water table

over a number of months. This transfer function can be evaluated at lags covering the three

month forecast horizon to define a model response characteristic, P, which specifies the

percentage of modelled effective rainfall that reaches the water table over this period.

Figure 7 a shows that this value ranges between $20-95 \%$ and that the relationship between

$402 \mathrm{P}$ and the derived model sensitivities can be approximated with an exponential curve $\left(\mathrm{R}^{2}=\right.$

0.79 ) that shows that as $P$ increases, the model sensitivity to rainfall also increases. The

404 permeability of each catchment is also likely to influence the sensitivity to rainfall. Indeed, a

405 closer fit is obtained if the model sensitivity is normalised by the catchment baseflow index

406 (BFI) (Figure 7b), taken from Marsh and Hannaford (2008), which defines the proportion of

407 effective rainfall that contributes to groundwater flow. Furthermore, the calibrated model

408 sensitivities also correlate well with an independent inference of the response time to

409 rainfall for each catchment estimated by the peak lead lag correlation $\left(\mathrm{CC}_{\max }\right)$ between

410 observed rainfall and de-seasonalised groundwater levels (Figure 7c), obtaining an $R^{2}$ of

4110.76.

412 
413 These findings demonstrate that the more pronounced the AquiMod lagging mechanism,

414 the less sensitive the three month simulations are to the choice of rainfall input. A similar

415 relationship between the sensitivity and an independent estimation of the peak

416 groundwater level response time to rainfall for each borehole, further indicates that the

417 catchment response time has a clear influence on the sensitivity, and therefore is also likely

418 to influence the skill of the forecasts. Consequently, the catchments have been split into

419 three equally sized groups representing slowly responding $\left(3 \leq C C_{\max } \leq 10\right)$, moderately

420 responding $\left(1 \leq C C_{\max } \leq 2\right)$ and quickly responding $\left(0 \leq C C_{\max } \leq 1\right)$ groundwater catchments.

421 These are indicated in Figure 7a-c by the circles, squares and triangles respectively and the

422 analyses in the subsequent sections are conducted using this grouping.

\subsection{Skill metrics}

424 For the purpose of this skill analysis the reforecasts have been subdivided into 36 different assessment groups for which an independent assessment of skill has been conducted. These

426 groups comprise the three categorical events, the four seasons and the three groundwater

427 response groups. Figure 8 shows the four skill measurements for all of the assessment

428 groups using the three different climate inputs.

429

430 The frequency bias ranges between 0.61 and 0.5 (Figure $8 \mathrm{a}$ ). In the summer (JJA), there is a

431 consistent under forecasting of below normal levels which is mainly offset by a positive

432 frequency bias for near normal events. The winter (DJF) forecasts show the opposite

433 pattern, under forecasting above normal events and over forecasting below normal events.

434 The fact that groundwater levels tend to peak in the winter and trough in the summer

435 indicates that there is a tendency for the forecasts to miss the groundwater level extremes. 
436 Indeed, on average, the above and below normal events show negative frequency biases of

$437-0.04$ and -0.11 respectively while the near normal event category shows a positive

438 frequency bias of 0.13 . This deficiency cannot be attributed to the driving rainfall data as all

439 assessment groups demonstrate that they are insensitive to this, except for the quickly

440 responding catchments in winter and autumn (SON) where using the best case observed

441 climate reduces the bias by approximately half. This insensitivity is also apparent in the

442 other skill metrics, indicating that the skill or lack of it stems more from the model than the

443 rainfall input in most situations.

444

445 Generally, the forecasts are more reliable when predicting above and below normal events

446 than near normal events, especially during winter and autumn and during the summer for

447 the quickly responding catchments (Figure 8b). Figure 9 shows the reliability diagrams for

448 the quickly responding catchments in winter. It can be seen that for the above and below

449 normal events the reliability curves follow the line of perfect reliability closely indicating

450 good consistency between the forecast probabilities and observed relative frequencies. In

451 contrast, there is a tendency for the forecasts to predict closer to base rate probabilities

452 (0.33) for the near normal events as indicated by the flat reliability curves which imply a lack

453 of forecast resolution. This is reflected in the ROC scores (Figure 8c) which are smaller on

454 average for the near normal events indicating that the forecasts are less efficient at

455 discriminating these events. Even so, all of the ROC scores obtained were greater than 0.5

456 showing that the number of hits exceeded the number of false alarms. The forecasts were

457 also able to discriminate below normal events with an average ROC score of 0.87 using the

458 downscaled GloSea climate which is particularly encouraging.

459 
460 The ROC scores also demonstrate a clear relationship with the catchment response times

461 where the less sensitive, slowly responding catchments have greater discrimination capacity

462 than the quickly responding catchments. However, this again appears to be an artefact of

463 the model skill rather than to do with the sensitivity to the rainfall input. Even so, the use of

464 observed climate consistently improves the discrimination capacity of the forecasts,

465 particularly for the quickly responding catchments where improvements of up to 0.14 are

466 shown.

467

468 From the 36 assessment groups, 35 return a positive CRPSS when using the climatology

469 rainfall input (Figure $8 d$ ). This indicates that even climatology yields forecasts that are a

470 better predictor of groundwater levels than a persistence forecast. Slightly fewer (31) of the

471 groups return a positive CRPSS using the observed rainfall and only 30 when using the

472 downscaled GloSea data. All suggest that the forecasts consistently outperform the

473 persistence approach.

474

3.3. Time-series analysis

475 Finally, the forecasts have been evaluated over three time periods which contain important

476 historical events including: i) the onset and persistence of below normal levels in 1996 and

477 1997, a period where many parts of the UK experienced groundwater drought; ii) the

478 subsequent transition back to normal levels in 1997 and 1998, broadly associated with the

479 end of the drought; and (iii) the onset and peak of above normal levels in the winter of

$4802000 / 2001$, a period where many boreholes recorded their highest ever levels and where

481 there was widespread groundwater flooding, particularly in the Chalk of south-east England.

482 Figure 10 shows the number of catchments in each response category that successfully 
483 forecast each event using the three different climate inputs. A forecast was only deemed a

484 success if all of the observed groundwater levels were contained within the forecast

485 uncertainty bounds as defined by the limits of the ensemble. In addition, Figure 11 displays

486 time-series plots for several of the study catchments over these events which have been

487 used to compare the observed groundwater levels (black dots) against the ensemble mean

488 forecasts (thick dashed lines) using the GloSea5 and observed rainfall inputs. The

489 uncertainty bounds (thin dashed lines) are also shown.

490

491 The forecasts were least effective at capturing the high levels of winter 2000/2001 when

492 using the downscaled GloSea and climatology rainfall, but showed significant improvements

493 when driven with observed data. This is demonstrated in Figure 11a where the observed

494 initial groundwater rise (time steps one to three) at the quickly responding New Red Lion

495 borehole, is only replicated by the ensemble mean forecast when using the observed rainfall

496 input. The forecast using the downscaled GloSea rainfall does not capture this due to

497 underestimating the seasonal rainfall by almost $130 \mathrm{~mm}$. Note that the GloSea forecast is

498 able to capture the peak groundwater level at the fourth time step. This is partly because

499 this corresponds to the one month ahead forecast, and therefore the model was initialised

500 at the above normal levels from the previous time step.

501

502 For the below normal levels of 1996 and 1997, the choice of climate has less impact on the

503 success rate which is demonstrated by the New Red Lion reforecast in Figure 11b. It can be

504 seen here that regardless of the rainfall input, the ensemble mean overestimates the

505 groundwater levels and the uncertainty bounds do not capture the gradual recession of the

506 hydrograph and even extend into the above normal range between time steps five and six. 
507 This insensitivity could be explained by the large soil moisture deficit that would likely

508 develop over this period. Therefore, the forecasts are much more reliant on the skill of the

509 model, which in this case does not capture the ground water discharge and subsequent

510 hydrograph recession adequately.

511

512 Some catchments, such as the quickly responding Bussels catchment (Figure 11c) did

513 demonstrate significant sensitivity to the rainfall input during this drought period. Here, it

514 can be seen that when using the observed rainfall, the ensemble mean follows the

515 persistent low groundwater levels closely, but when using the downscaled GloSea rainfall,

516 the ensemble mean forecast actually predicts a sharp rise in groundwater level almost back

517 to normal conditions (time steps seven to nine) due to the downscaled GloSea forecast

518 overestimating rainfall by $100 \mathrm{~mm}$ for this period.

519

520 The highest overall success rates using the downscaled GloSea inputs were recorded for the

521 return to normal levels in 1997 and 1998. For the moderately responding Rockley

522 observation borehole (Figure 11d), it can be seen that the two ensemble mean forecasts

523 using the GloSea and observed rainfall inputs are similar. Furthermore, both capture all of

524 the observations in their uncertainty bounds which was observed for most of the

525 catchments for this period.

526 4. Discussion

527 This study has demonstrated that skilful seasonal forecasts of groundwater levels at

528 observation boreholes can be generated by using seasonal weather forecasts to drive

529 parsimonious conceptual groundwater models. The forecasts were proficient at 
530 discriminating between below, near and above normal future groundwater levels and they

531 consistently outperformed a reference persistence forecast system. They also demonstrated

532 good reliability, particularly for the seasonal forecasts of spring groundwater levels. These

533 positive attributes have also been demonstrated for the quickly responding catchments,

534 indicating that the skill can extend beyond the peak response time of these groundwater

535 systems.

536

537 The skill of the forecasts originates from a combination of the driving climate data, the

538 groundwater models and the initial groundwater level condition. For those catchments

539 where groundwater levels respond more slowly to rainfall, the groundwater models and the

540 initial conditions have a stronger influence on the forecast skill than the rainfall input.

541 However, there is no clear indication that the sensitivity to the rainfall input directly affects

542 the forecast skill. Rather, the relationship between groundwater level response time to

543 rainfall and forecast skill appears to be primarily controlled by the groundwater model

544 efficiency. Indeed, when conducting this work, we could find no apparent correlation

545 between skill and geographical location like, for example, the work of Svensson et al. (2015).

546 However, we suggest that with a larger sample size of boreholes, and by evaluating the

547 forecast skill at longer lead times, where meteorological driving data plays a more crucial

548 role in the forecast skill, such relationships may become more apparent. Indeed, while all of

549 the response groups demonstrated forecast skill, it remains to be seen at what lead time

550 this skill diminishes.

551

552 The origin of forecast skill also changes as a response to antecedent hydro-meteorological

553 conditions. Here, it was found that when a large soil moisture deficit is developed during the 
554 model spin up and initialisation, the subsequent forecasts are less sensitive to the rainfall

555 input. As such, we noted that for the summer forecasts, the skill derives mainly from the

556 groundwater models and their internal hydrogeological memory. This has potential

557 implications because some of the models have shown deficiencies, such as poor

558 representation of the hydrograph recession, which materialised as forecast errors. Some of

559 these deficiencies are likely to result from imperfect model calibration, errors in the

560 meteorological input data and observed groundwater levels, or from inadequacies of the

561 model structure and parameters. In this study, no account of input error was made, but we

562 did acknowledge some of the model uncertainties by using an equifinality of acceptable

563 model structures and parameter sets. Of course, this approach in itself may also propagate

564 forecasting errors. For example, the choice of the NSE as a measure of model likelihood was

565 subjective, and as with any objective function, is subject to undesirable properties that are

566 likely to manifest themselves as modelling errors (Smith et al., 2008). There is also evidence

567 that model appropriateness depends strongly on hydro-climatic conditions (Herman et al.,

568 2013) and that it may be beneficial to develop better suited limits of acceptability which can

569 be relaxed dynamically as a mean to implicitly account for input errors (Liu et al., 2009).

570

571 In contrast to the forecasts issued following dry conditions, during winter, when the soil is

572 generally more saturated, the forecasts are more sensitive to the driving rainfall data, and

573 as such the meteorological forecasts play a more crucial role in the skill of the groundwater

574 level forecasts. The winter forecasts using the downscaled GloSea and climatology rainfall

575 inputs both consistently outperformed the persistence approach, although it should be

576 noted that using the downscaled GloSea5 rainfall data showed no significant improvement

577 over using the site climatology inputs, and in some cases showed to be a worse rainfall 
578 predictor. This is perhaps not surprising given that UK rainfall has complex spatio-temporal

579 signatures that make deriving robust downscaling transformations difficult. Certainly, the

580 linear downscaling model employed showed to be inadequate for some sites, and improving

581 this should be a high priority for improving site-specific hydrological forecasts like these.

582 However, it may be possible to improve this using more sophisticated non-linear

583 downscaling and post processing techniques which have shown to be effective for medium-

584 range ensemble streamflow forecasts (Verkade et al., 2013). Further data assimilation could

585 also provide enhancements in skill through dynamic updating of state variables and forecast

586 errors, although to date there is limited evidence that this is useful for seasonal or

587 groundwater level forecasting applications (Liu et al., 2012). There are also other seasonal

588 weather forecasting models which could be used for these types of applications, such as the

589 System 4 from the European Centre for Medium-range Weather Forecasts (ECMWF) which

590 has shown "marginally useful" degrees of reliability over Northern Europe (Weisheimer and

591 Palmer, 2014).

592

593 It is important to note that the interpretation of skill in this study is primarily based on

594 analysis of the verification metrics and by comparing the forecasts to the benchmark results.

595 Pappenberger et al. (2015) compared a range of benchmarks for medium-range river flow

596 forecasting and they note that the best benchmarks are the ones that are hardest to beat.

597 While considerable effort was made to select appropriate benchmarks and avoid reporting

598 "naïve" skill, it should be noted that the persistence benchmark used is less skilful for those

599 boreholes that exhibit significant inter-annual groundwater level fluctuations, and so there

600 is likely to be positive bias in the CRPSS reported for the more slowly responding

601 catchments. Certainly, an equivalent thorough examination of benchmark performance to 
602 that of Pappenberger et al. (2015) is also needed for seasonal groundwater level

603 forecasting.

604

605 It is also important to consider that, the verification metrics used in this study only give an

606 average indication of the forecast's ability to reliably discriminate between the occurrence

607 of below, near and above normal levels over the 14-year reforecast sequence. When looking

608 at the extreme 2000/2001 high groundwater level event specifically, only two of the 21

609 groundwater level forecasts were able to capture it within their uncertainty bounds when

610 using the downscaled GloSea and climatology inputs. For the 1996/1997 drought period, the

611 timing of the return to normal conditions could only be predicted when using observed

612 rainfall data. This is an important issue, as it is arguably extreme events like these that, if

613 foreseeable, would provide the most economic, environmental and societal benefit. That of

614 course is not to say that these forecasts are not useful; on the contrary the Environment

615 Agency in England, for example, routinely use measures of aquifer levels relative to normal

616 conditions to inform agricultural communities about future prospects for spray irrigation

617 and this approach can be used to help aid decision making processes for these needs. It

618 does however mean that if we wish to forecast the initiation or end of extreme events on a

619 seasonal time scale at the catchment or borehole resolution, then further enhancements in

620 the skill and the use of seasonal rainfall forecasts are required.

621 5. Conclusions

622 Using seasonal weather forecasts to drive 21 conceptual groundwater models, this study

623 has shown that skilful seasonal forecasts of groundwater levels at observation boreholes

624 can be generated up to three months into the future. Site-specific groundwater level 
625 response characteristics to rainfall result in contrasting sensitivities to the driving rainfall

626 input across the study catchments. These sensitivities have also shown to be strongly

627 controlled by prevailing weather conditions, where dry conditions tend to result in forecasts

628 that are strongly controlled by the groundwater model, and wet conditions result in

629 forecasts that are much more reliant on good driving rainfall data. This has important

630 implications for where the skill or lack of it derives from, and more importantly, where

631 future improvements can be made. There are clearly issues with correctly forecasting

632 extreme groundwater levels which are primarily due to lack of skill in the driving rainfall

633 data. In particular it is recommended that future work should focus these aspects:

634 1. Investigate the best practice for data assimilation, downscaling and post processing

3. Examine the maximum skilful forecast lead time for different aquifers in relation to their response characteristics to rainfall.

\section{Acknowledgements}

641 Mackay, Jackson and Ward were supported by core science funds of the Natural

642 Environment Research Council (NERC) British Geological Survey's (BGS) Groundwater

643 Science and Environmental Modelling Directorates. Scaife was supported by the Joint

644 DECC/Defra Met Office Hadley Centre Climate Programme (GA01101) and Brookshaw was

645 supported by the UK Public Weather Service research program. The groundwater level data

646 for this study were taken from the NERC BGS National Groundwater Level Archive. The

647 climate data were made available by the NERC Centre for Ecology and Hydrology, and the 
UK Met-Office. Mackay, Jackson and Ward publish with the permission of the Executive

649

Director of the British Geological Survey.

650

651

652

653

654

655

656

657

658

659

660

661

662

663

664

665

666

667

668

669

670

671

672

673

674

675

676

677

678

679

680

681

682

683

\section{References}

Adamowski, J. \& Chan, H. F. 2011. A wavelet neural network conjunction model for ground water level forecasting. Journal of Hydrology, 407, 2840.

Addor, N., Jaun, S., Fundel, F. \& Zappa, M. 2011. An operational hydrological ensemble prediction system for the city of Zurich (Switzerland): skill, case studies and scenarios. Hydrol. Earth Syst. Sci., 15, 2327-2347.

Alley, W. M., Healy, R. W., LaBaugh, J. W. \& Reilly, T. E. 2002. Flow and Storage in Groundwater Systems. Science, 296, 1985-1990.

Behzad, M., Asghari, K. \& Coppola, E. 2010. Comparative Study of SVMs and ANNs in Aquifer Water Level Prediction. Journal of Computing in Civil Engineering, 24, 408-413.

Bell, V. A., Davies, H. N., Kay, A. L., Marsh, T. J., Brookshaw, A. \& Jenkins, A. 2013. Developing a large-scale water-balance approach to seasonal forecasting: application to the 2012 drought in Britain. Hydrological Processes, 27, 3003-3012.

Bennett, N. D., Croke, B. F. W., Guariso, G., Guillaume, J. H. A., Hamilton, S. H., Jakeman, A. J., Marsili-Libelli, S., Newham, L. T. H., Norton, J. P., Perrin, C., Pierce, S. A., Robson, B., Seppelt, R., Voinov, A. A., Fath, B. D. \& Andreassian, $V$. 2013. Characterising performance of environmental models. Environmental Modelling \& Software, 40, 1-20.

Beven, K. 2006. A manifesto for the equifinality thesis. Journal of Hydrology, 320, 18-36.

Beven, K. \& Binley, A. 1992. The future of distributed models: Model calibration and uncertainty prediction. Hydrological Processes, 6, 279298.

Beven, K. \& Binley, A. 2013. GLUE: 20 years on. Hydrological Processes, n/a$\mathrm{n} / \mathrm{a}$.

Bloomfield, J. P. \& Marchant, B. P. 2013. Analysis of groundwater drought building on the standardised precipitation index approach. Hydrol. Earth Syst. Sci., 17, 4769-4787.

Boorman, D. B., Hollis, J. M. \& Lilly, A. 1995. Report No. 126 Hydrology of soil types: a hydrologically-based classification of the soils of the United Kingdom. Institute of Hydrology, Wallingford, UK. 
684

685

686

687

688

689

690

691

692

693

694

695

696

697

698

699

700

701

702

703

704

705

706

707

708

709

710

711

712

713

714

715

716

717

718

719

720

721

722

723

Buizza, R. 2008. The value of probabilistic prediction. Atmospheric Science Letters, 9, 36-42.

Cloke, H. L. \& Pappenberger, F. 2009. Ensemble flood forecasting: A review. Journal of Hydrology, 375, 613-626.

Cuo, L., Pagano, T. C. \& Wang, Q. J. 2011. A Review of Quantitative Precipitation Forecasts and Their Use in Short- to Medium-Range Streamflow Forecasting. Journal of Hydrometeorology, 12, 713-728.

Daliakopoulos, I. N., Coulibaly, P. \& Tsanis, I. K. 2005. Groundwater level forecasting using artificial neural networks. Journal of Hydrology, 309, 229-240.

Eltahir, E. A. B. \& Yeh, P. J. F. 1999. On the asymmetric response of aquifer water level to floods and droughts in Illinois. Water Resources Research, 35, 1199-1217.

Field, M. 1983. The meteorological office rainfall and evaporation calculation system - MORECS. Agricultural Water Management, 6, 297-306.

Herman, J. D., Reed, P. M. \& Wagener, T. 2013. Time-varying sensitivity analysis clarifies the effects of watershed model formulation on model behavior. Water Resources Research, 49, 1400-1414.

Huntingford, C., Marsh, T., Scaife, A. A., Kendon, E. J., Hannaford, J., Kay, A. L., Lockwood, M., Prudhomme, C., Reynard, N. S., Parry, S., Lowe, J. A., Screen, J. A., Ward, H. C., Roberts, M., Stott, P. A., Bell, V. A., Bailey, M., Jenkins, A., Legg, T., Otto, F. E. L., Massey, N., Schaller, N., Slingo, J. \& Allen, M. R. 2014. Potential influences on the United Kingdom's floods of winter 2013/14. Nature Clim. Change, 4, 769-777.

Jakeman, A. J., Letcher, R. A. \& Norton, J. P. 2006. Ten iterative steps in development and evaluation of environmental models. Environmental Modelling \& Software, 21, 602-614.

Jolliffe, I. T. \& Stephenson, D. B. 2012. Forecast Verification: A Practitioner's Guide in Atmospheric Science. Wiley, Chichester, UK.

Lanen, H. A. J. \& Peters, E. 2000. Definition, Effects and Assessment of Groundwater Droughts. In: VOGT, J. \& SOMMA, F. (eds.) Drought and Drought Mitigation in Europe. Springer Netherlands.

Lewis, J. M. 2005. Roots of Ensemble Forecasting. Monthly Weather Review, 133, 1865-1885.

Liu, Y., Freer, J., Beven, K. \& Matgen, P. 2009. Towards a limits of acceptability approach to the calibration of hydrological models: Extending observation error. Journal of Hydrology, 367, 93-103.

Liu, Y., Weerts, A. H., Clark, M., Hendricks Franssen, H. J., Kumar, S., Moradkhani, H., Seo, D. J., Schwanenberg, D., Smith, P., van Dijk, A. I. J. M., van Velzen, N., He, M., Lee, H., Noh, S. J., Rakovec, O. \& Restrepo, P. 
724

725

726

727

728

729

730

731

732

733

734

735

736

737

738

739

740

741

742

743

744

745

746

747

748

749

750

751

752

753

754

755

756

757

758

759

760

761

2012. Advancing data assimilation in operational hydrologic forecasting: progresses, challenges, and emerging opportunities. Hydrol. Earth Syst. Sci., 16, 3863-3887.

Lorenz, E. N. 1963. Deterministic Nonperiodic Flow. Journal of the Atmospheric Sciences, 20, 130-141.

Mackay, J. D., Jackson, C. R. \& Wang, L. 2014. A lumped conceptual model to simulate groundwater level time-series. Environmental Modelling \& Software, 61, 229-245.

MacLachlan, C., Arribas, A., Peterson, K. A., Maidens, A., Fereday, D., Scaife, A. A., Gordon, M., Vellinga, M., Williams, A., Comer, R. E., Camp, J., Xavier, P. \& Madec, G. 2014. Global Seasonal forecast system version 5 (GloSea5): a high-resolution seasonal forecast system. Quarterly Journal of the Royal Meteorological Society, n/a-n/a.

Maheswaran, R. \& Khosa, R. 2013. Long term forecasting of groundwater levels with evidence of non-stationary and nonlinear characteristics. Computers \& Geosciences, 52, 422-436.

Maier, H. R. \& Dandy, G. C. 2000. Neural networks for the prediction and forecasting of water resources variables: a review of modelling issues and applications. Environmental Modelling \& Software, 15, 101-124.

Marsh, T., Cole, G. \& Wilby, R. 2007. Major droughts in England and Wales, 1800-2006. Weather, 62, 87-93.

Marsh, T. J. \& Hannaford, J. 2008. UK Hydrometric Register. Centre for Ecology and Hydrology, Wallingford, UK.

Mendicino, G., Senatore, A. \& Versace, P. 2008. A Groundwater Resource Index (GRI) for drought monitoring and forecasting in a mediterranean climate. Journal of Hydrology, 357, 282-302.

Mishra, A. K. \& Singh, V. P. 2010. A review of drought concepts. Journal of Hydrology, 391, 202-216.

Monteith, J. L. \& Unsworth, M. H. 2008. Principles of Environmental Physics: Third Edition. Elsevier, London, UK.

Moore, R. J. \& Bell, V. A. 1999. Incorporation of groundwater losses and well level data in rainfall-runoff models illustrated using the PDM. Hydrol. Earth Syst. Sci., 6, 25-38.

Murphy, A. H. 1973. A New Vector Partition of the Probability Score. Journal of Applied Meteorology, 12, 595-600.

Nash, J. E. \& Sutcliffe, J. V. 1970. River flow forecasting through conceptual models part I - A discussion of principles. Journal of Hydrology, 10, 282290. 
762

763

764

765

766

767

768

769

770

771

772

773

774

775

776

777

778

779

780

781

782

783

784

785

786

787

788

789

790

791

792

793

794

795

796

797

798

799

800

Nourani, V., Mogaddam, A. A. \& Nadiri, A. O. 2008. An ANN-based model for spatiotemporal groundwater level forecasting. Hydrological Processes, 22, 5054-5066.

Pappenberger, F., Beven, K. J., Hunter, N. M., Bates, P. D., Gouweleeuw, B. T., Thielen, J. \& de Roo, A. P. J. 2005. Cascading model uncertainty from medium range weather forecasts (10 days) through a rainfall-runoff model to flood inundation predictions within the European Flood Forecasting System (EFFS). Hydrol. Earth Syst. Sci., 9, 381-393.

Pappenberger, F., Ramos, M. H., Cloke, H. L., Wetterhall, F., Alfieri, L., Bogner, K., Mueller, A. \& Salamon, P. 2015. How do I know if my forecasts are better? Using benchmarks in hydrological ensemble prediction. Journal of Hydrology, 522, 697-713.

Partal, T. \& Kişi, Ö. 2007. Wavelet and neuro-fuzzy conjunction model for precipitation forecasting. Journal of Hydrology, 342, 199-212.

Perry, M., Hollis, D. \& Elms, M. 2009. The Generation of Daily Gridded Datasets of Temperature and Rainfall for the UK. Exeter, UK.

Pinault, J. L., Amraoui, N. \& Golaz, C. 2005. Groundwater-induced flooding in macropore-dominated hydrological system in the context of climate changes. Water Resources Research, 41, W05001.

Sahu, B. K. 2003. Time Series Modelling in Earth Sciences. A.A. Balkema, Netherlands.

Scaife, A. A., Arribas, A., Blockley, E., Brookshaw, A., Clark, R. T., Dunstone, N., Eade, R., Fereday, D., Folland, C. K., Gordon, M., Hermanson, L., Knight, J. R., Lea, D. J., MacLachlan, C., Maidens, A., Martin, M., Peterson, A. K., Smith, D., Vellinga, M., Wallace, E., Waters, J. \& Williams, A. 2014. Skillful long-range prediction of European and North American winters. Geophysical Research Letters, 41, 2014 GL059637.

Smith, P., Beven, K. J. \& Tawn, J. A. 2008. Informal likelihood measures in model assessment: Theoretic development and investigation. Advances in Water Resources, 31, 1087-1100.

Sperna Weiland, F. C., van Beek, L. P. H., Kwadijk, J. C. J. \& Bierkens, M. F. P. 2010. The ability of a GCM-forced hydrological model to reproduce global discharge variability. Hydrol. Earth Syst. Sci., 14, 1595-1621.

Sreekanth, P. D., Geethanjali, N., Sreedevi, P. D., Ahmend, S., Ravi Kumar, N. \& Kamala Jayanthi, P. D. 2009. Forecasting groundwater level using artificial neural networks Current Science, 96, 933-939.

Sukhija, B. S. 2008. Adaptation to climate change: strategies for sustaining groundwater resources during droughts. Geological Society, London, Special Publications, 288, 169-181. 
801

802

803

804

805

806

807

808

809

810

811

812

813

814

815

816

817

818

819

820

821

822

823

824

825

826

827

828

829

830

831

832

833

834

835

836

837

838

839

Suryanarayana, C., Sudheer, C., Mahammood, V. \& Panigrahi, B. K. 2014. An integrated wavelet-support vector machine for ground water level prediction in Visakhapatnam, India. Neurocomputing, 145, 324-335.

Svensson, C., Brookshaw, A., Scaife, A. A., Bell, V. A., Mackay, J. D., Jackson, C. R., Hannaford, J., Davies, H. N., Arribas, A. \& Stanley, S. 2015. Long-range forecasts of UK winter hydrology. Environmental Research Letters, 10, 064006.

Taormina, R., Chau, K.-w. \& Sethi, R. 2012. Artificial neural network simulation of hourly groundwater levels in a coastal aquifer system of the Venice lagoon. Engineering Applications of Artificial Intelligence, 25, 1670-1676.

Trichakis, I. C., Nikolos, I. K. \& Karatzas, G. P. 2009. Optimal selection of artificial neural network parameters for the prediction of a karstic aquifer's response. Hydrological Processes, 23, 2956-2969.

Tsanis, I. K., Coulibaly, P. \& Daliakopoulos, I. N. 2008. Improving groundwater level forecasting with a feedforward neural network and linearly regressed projected precipitation. Journal of Hydroinformatics, 10, 317330.

Upton, K. A. \& Jackson, C. R. 2011. Simulation of the spatio-temporal extent of groundwater flooding using statistical methods of hydrograph classification and lumped parameter models. Hydrological Processes, 25, 1949-1963.

Vapnik, V. N. 1999. An overview of statistical learning theory. Neural Networks, IEEE Transactions on, 10, 988-999.

Verkade, J. S., Brown, J. D., Reggiani, P. \& Weerts, A. H. 2013. Post-processing ECMWF precipitation and temperature ensemble reforecasts for operational hydrologic forecasting at various spatial scales. Journal of Hydrology, 501, 73-91.

Weisheimer, A. \& Palmer, T. N. 2014. On the reliability of seasonal climate forecasts.

Xiong, L. \& O'Connor, K. M. 2008. An empirical method to improve the prediction limits of the GLUE methodology in rainfall-runoff modeling. Journal of Hydrology, 349, 115-124.

Ying, Z., Wenxi, L., Haibo, C. \& Jiannan, L. 2014. Comparison of three forecasting models for groundwater levels: a case study in the semiarid area of west Jilin Province, China. Journal of Water Supply, 63, 671-683.

Yoon, H., Jun, S.-C., Hyun, Y., Bae, G.-O. \& Lee, K.-K. 2011. A comparative study of artificial neural networks and support vector machines for predicting groundwater levels in a coastal aquifer. Journal of Hydrology, 396, 128138. 
840

841

842

843

844

845

846

847

848

849

850

851

852

853

854

855

856

857

858

859

860

861

862

863

864

865

866

867

868

869

870
Yossef, N. C., van Beek, L. P. H., Kwadijk, J. C. J. \& Bierkens, M. F. P. 2012. Assessment of the potential forecasting skill of a global hydrological model in reproducing the occurrence of monthly flow extremes. Hydrol. Earth Syst. Sci., 16, 4233-4246.

Yossef, N. C., Winsemius, H., Weerts, A., van Beek, R. \& Bierkens, M. F. P. 2013. Skill of a global seasonal streamflow forecasting system, relative roles of initial conditions and meteorological forcing. Water Resources Research, 49, 4687-4699.

Zappa, M., Beven, K. J., Bruen, M., Cofiño, A. S., Kok, K., Martin, E., Nurmi, P., Orfila, B., Roulin, E., Schröter, K., Seed, A., Szturc, J., Vehviläinen, B., Germann, U. \& Rossa, A. 2010. Propagation of uncertainty from observing systems and NWP into hydrological models: COST-731 Working Group 2. Atmospheric Science Letters, 11, 83-91.

Zappa, M., Jaun, S., Germann, U., Walser, A. \& Fundel, F. 2011. Superposition of three sources of uncertainties in operational flood forecasting chains. Atmospheric Research, 100, 246-262. 
871

872

873

874

875

876

877

878

879

880

881

882

883

884

885

886

887

888

889

890

891

892 


\section{Figures}

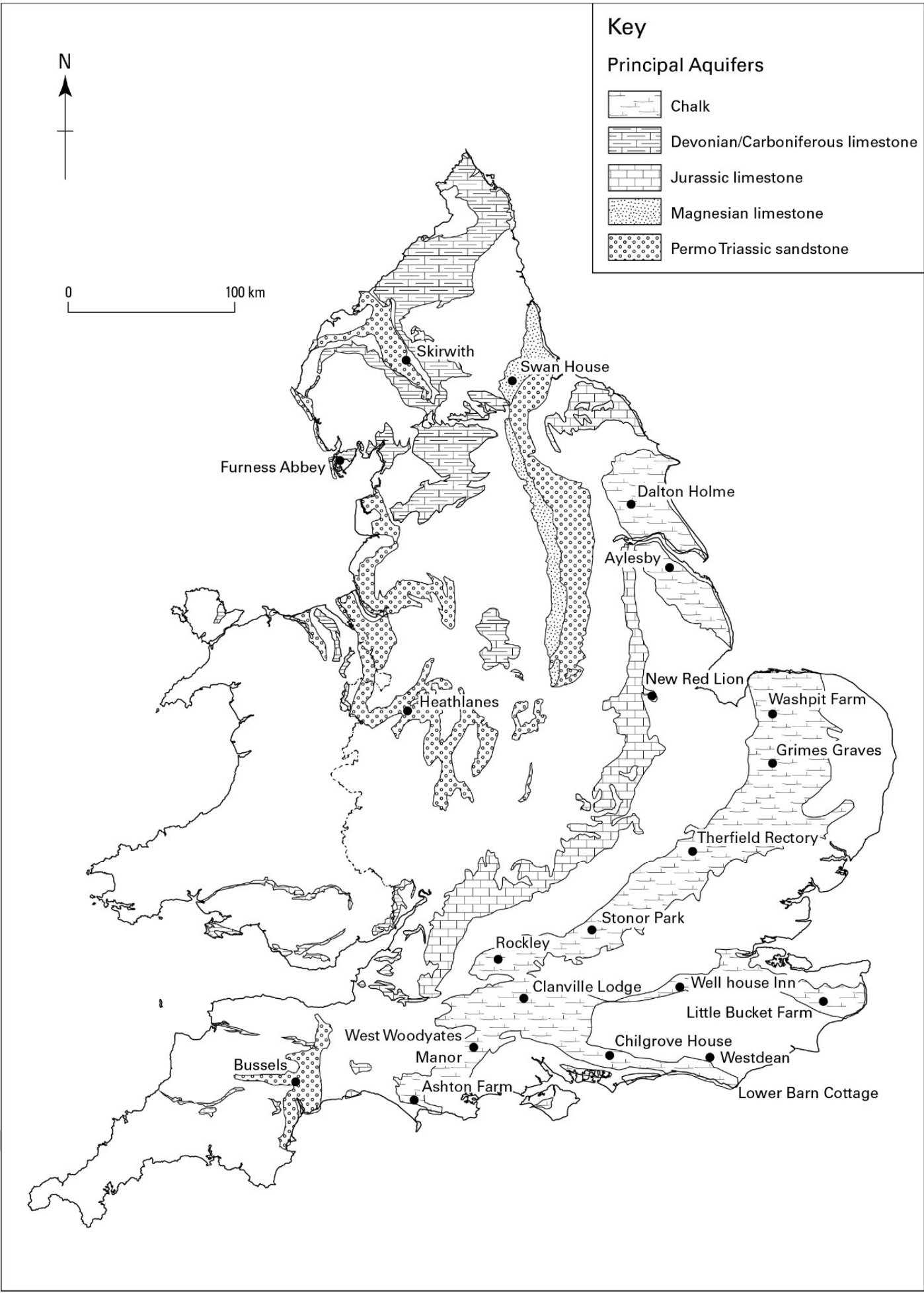

Figure 1: Observation borehole locations across the principal aquifers of the UK. 


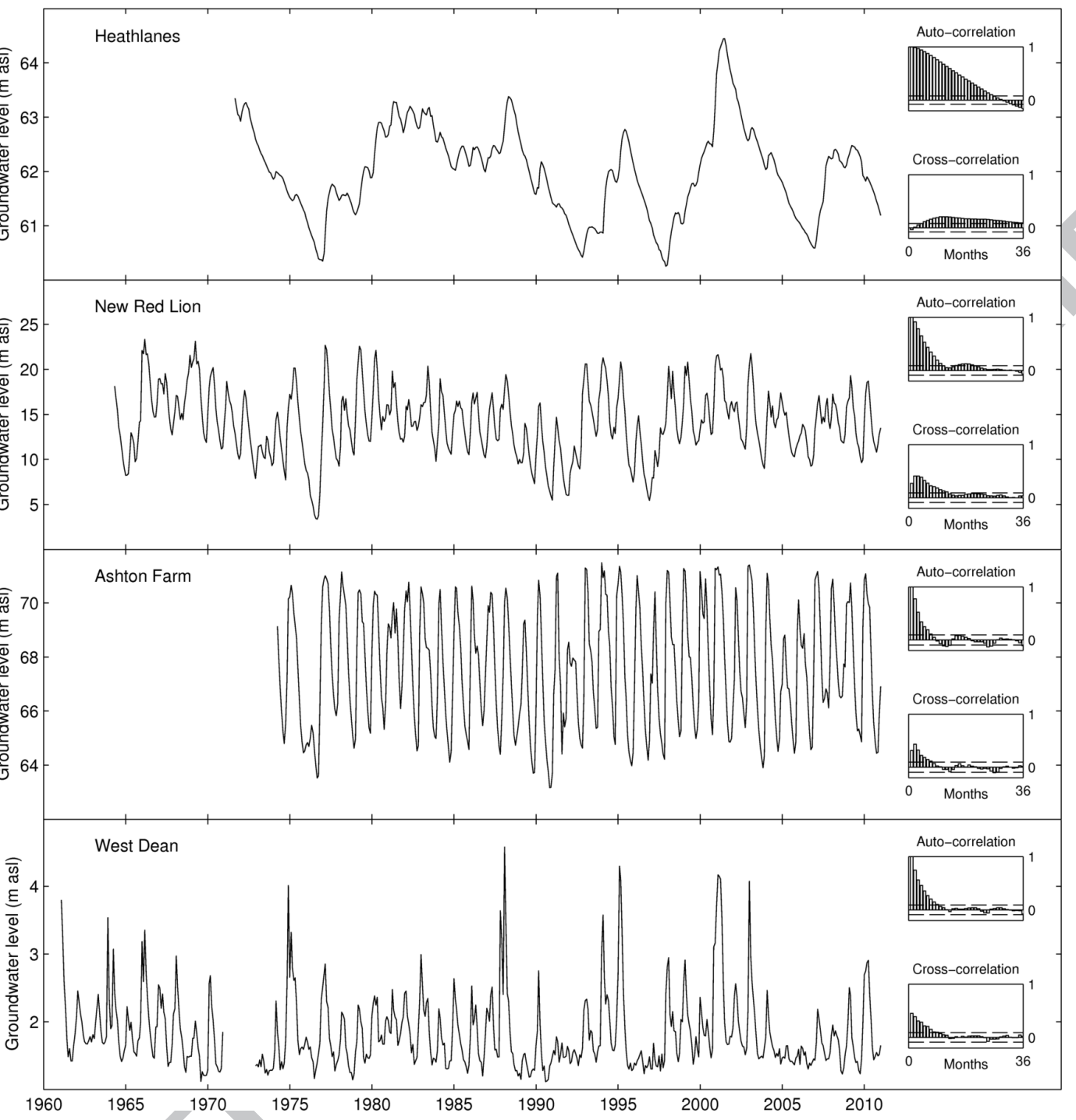

897

Figure 2: Groundwater level time-series with groundwater level auto-correlation and rainfall-groundwater level

899 cross-correlation plots. Note that the vertical scales vary across the plots. 


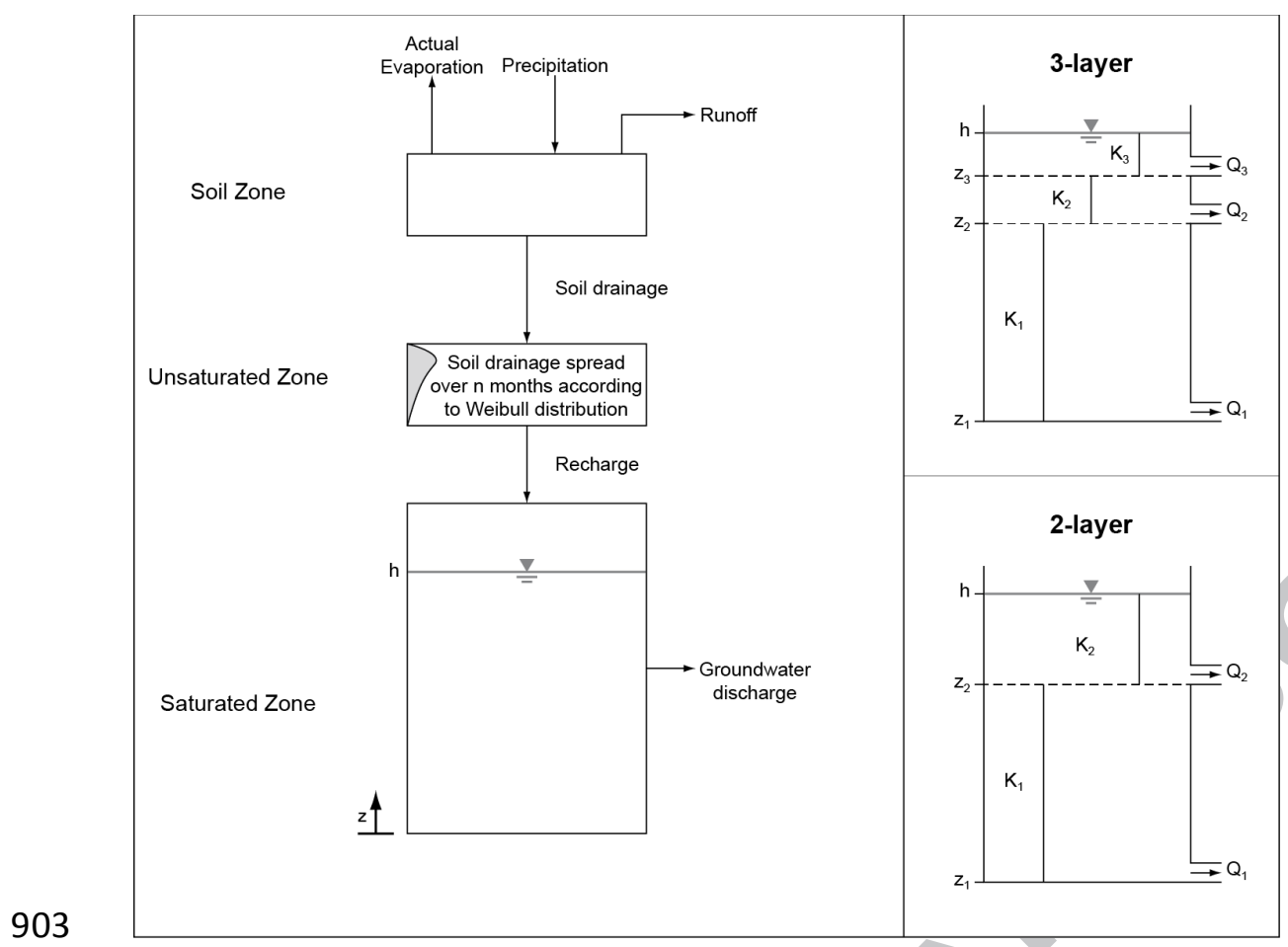

904 Figure 3: Schematic of generalised AquiMod model structure (left) and different saturated zone component

905 structures used in this study (right) after Mackay et al. (2014).

906

907

908

909 


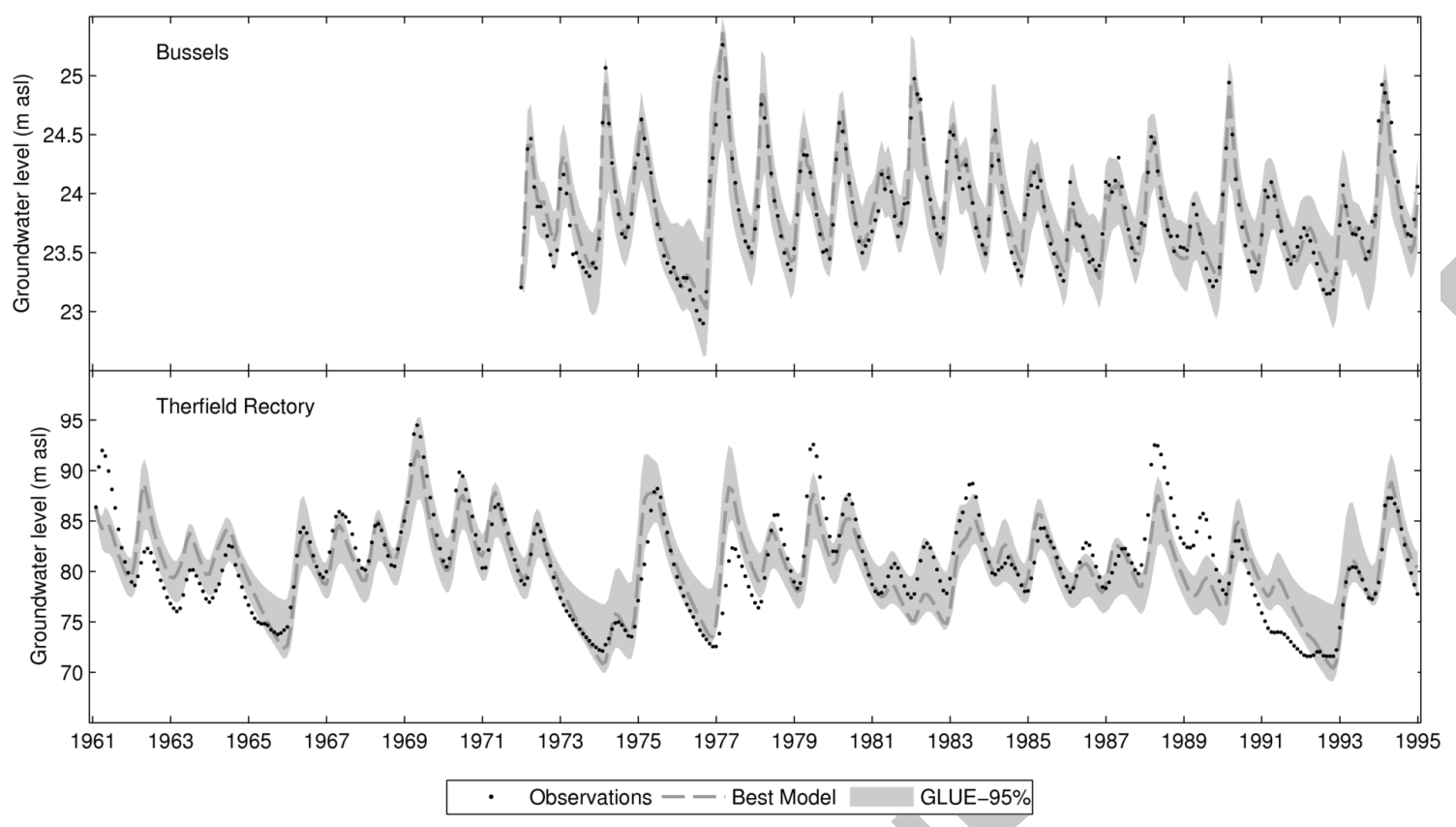

910

Figure 4: Calibration period simulations and observations for the Bussels and the Therfield Rectory observation

912 boreholes.

913 

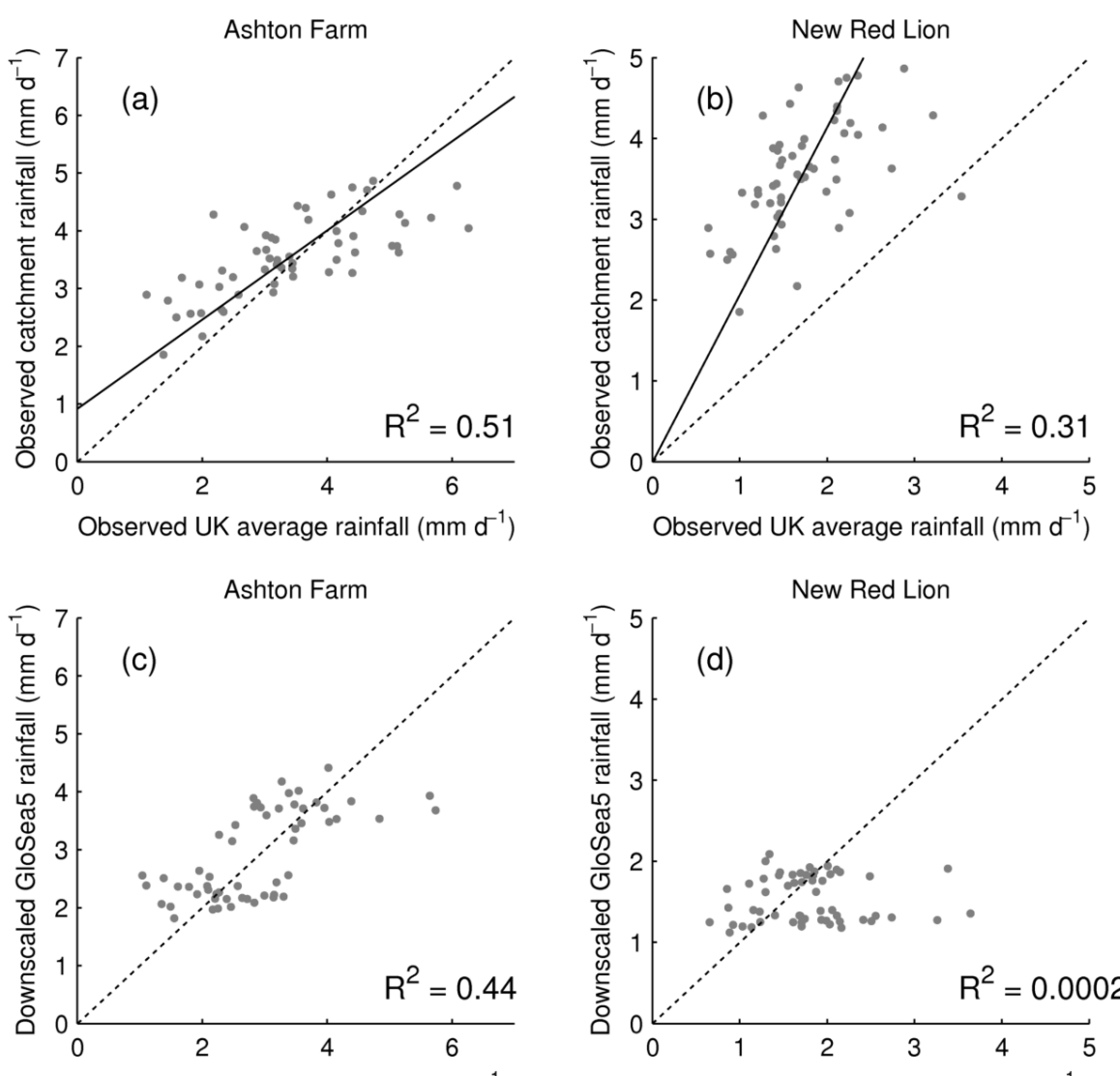
Observed catchment rainfall $\left(\mathrm{mm} \mathrm{d}^{-1}\right)$

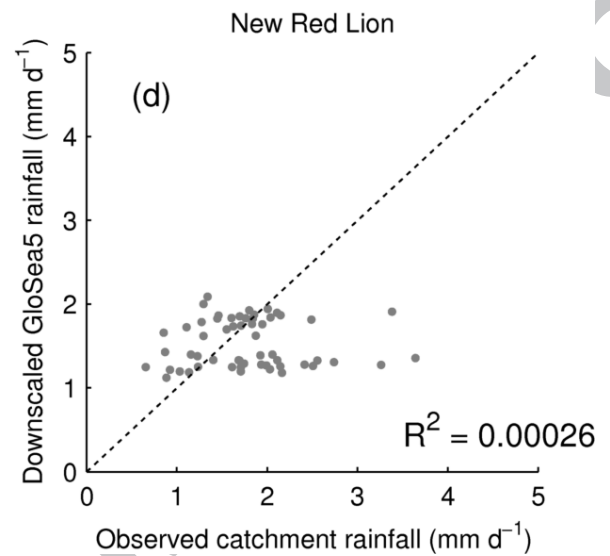

915 Figure 5: Linear regression models (solid black lines) fitted to downscale seasonal rainfall from UK average to

916 catchment scale for the Ashton Farm (a) and New Red Lion (b) observation boreholes. The resulting correlation

917 between the downscaled GloSea5 rainfall forecasts and the observed catchment rainfall is also shown for the

918 Ashton Farm (c) and New Red Lion (d) observation boreholes. 

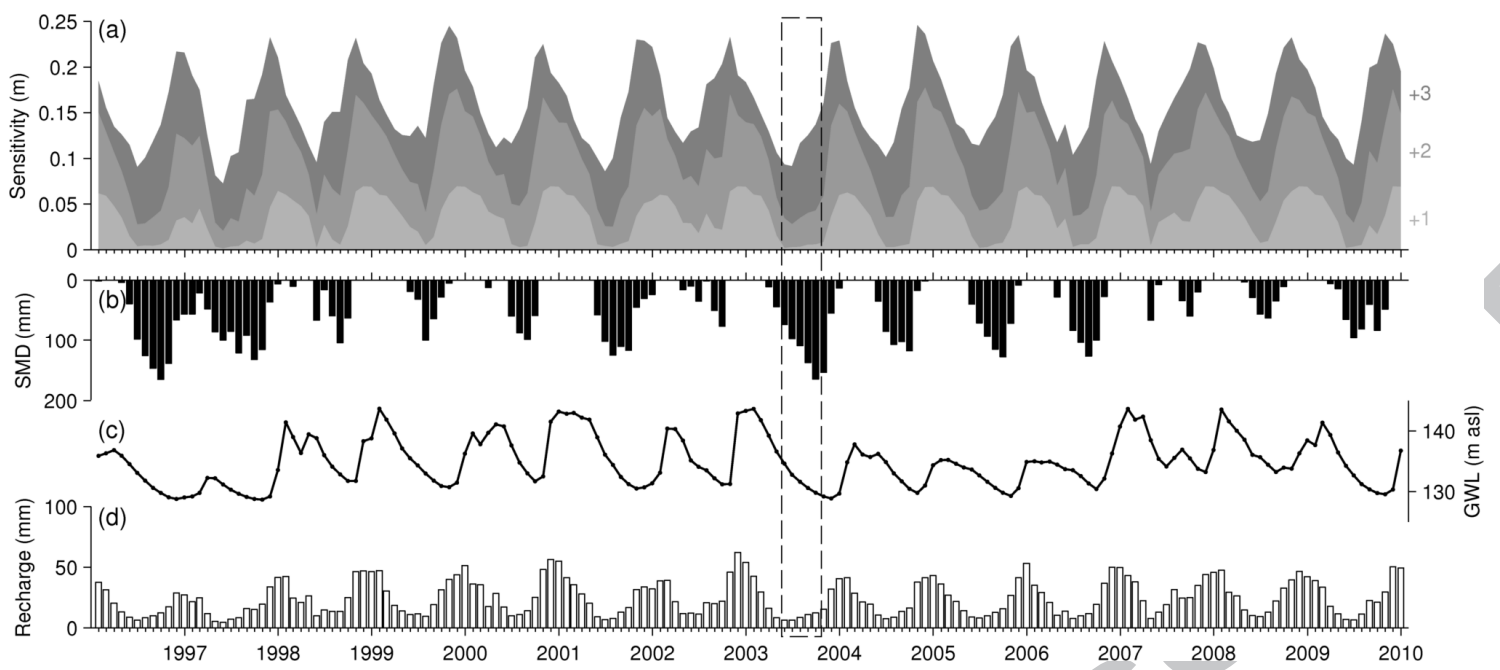

Figure 6: Calculated monthly sensitivity to climate inputs for one, two and three month forecasts (a) ; Soil recharge (d).
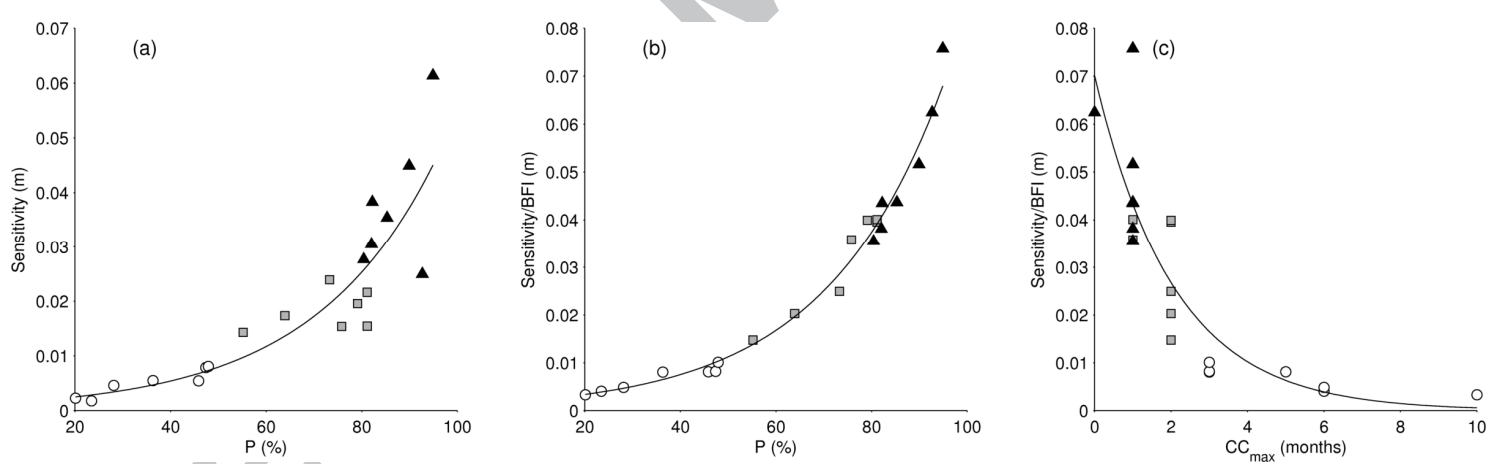

927 Figure 7: Model response characteristic, $P$, against the derived model sensitivity (a); P against the sensitivity

928 normalised with respect to the BFI (b); and the peak lead lag correlation between observed rainfall and deseasonalised groundwater levels, $C C_{\max }$, against the sensitivity normalised with respect to the $B F I$ (c) for the 21 catchment models. All data points are arranged into slowly (circles), moderately (squares) and quickly (triangles) responding catchments. 


\section{ACCEPTED MANUSCRIPT}
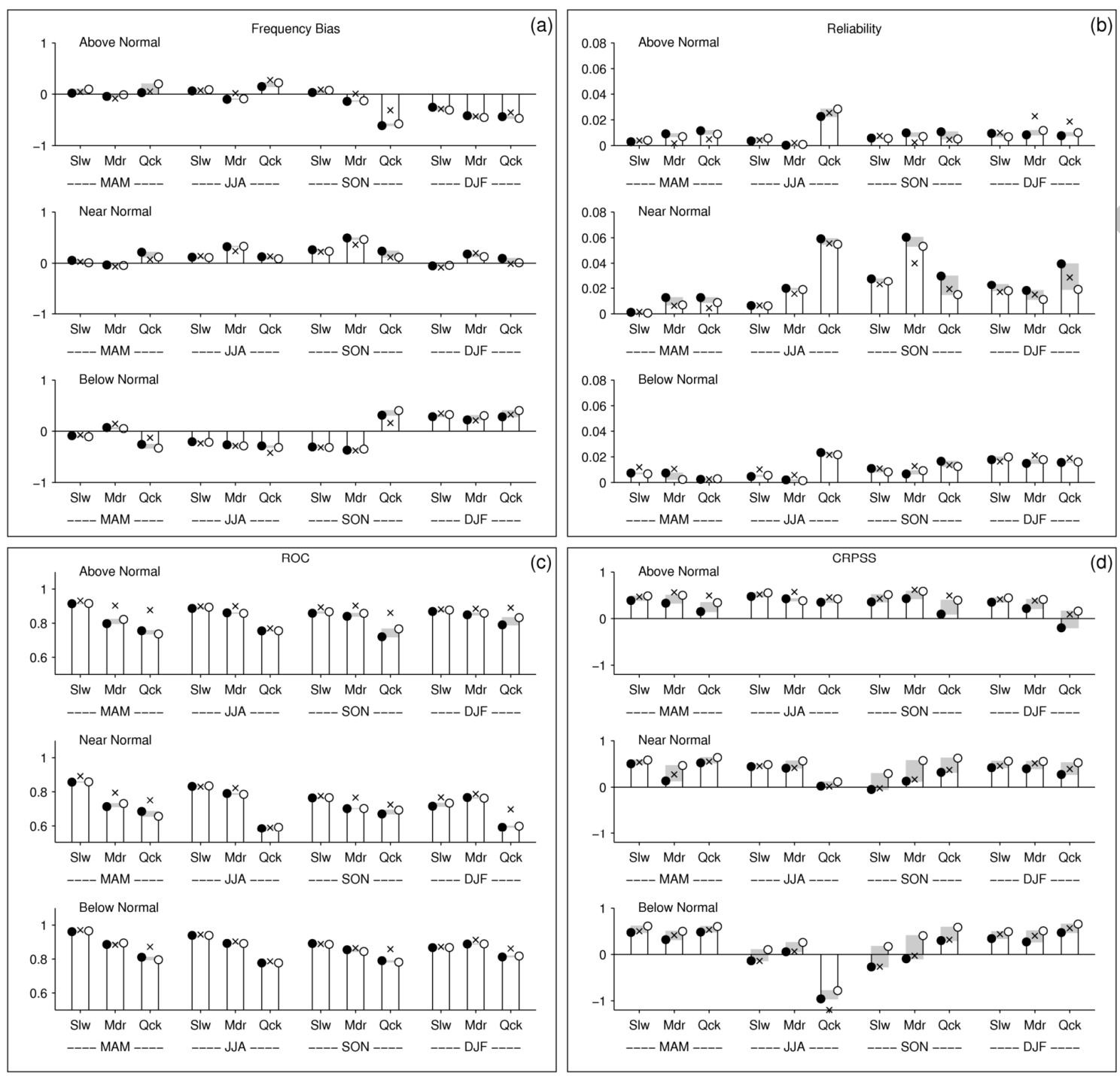

934

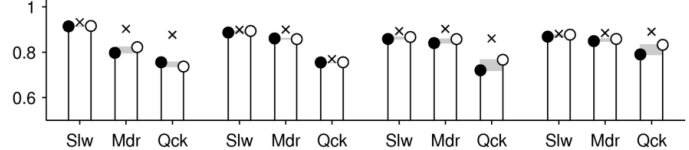

Slw Mdr Qck Slw Mdr Qck Slw Mdr Qck Slw Mdr Qck

---- MAM ---- ---- JJA ---- ---- SON ---- ---- DJF ----

Near Normal

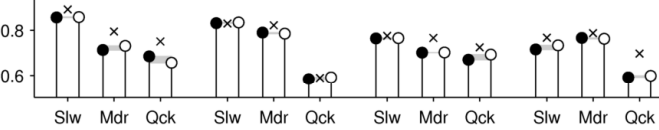

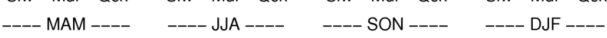

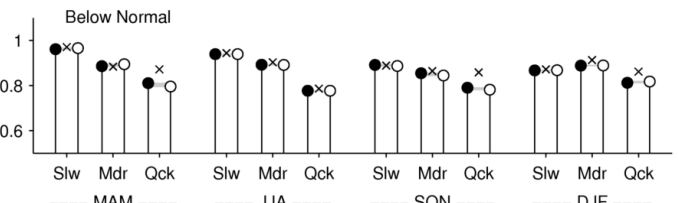

- GloSea

Climatology

GloSea-climatology range

935

Figure 8: Frequency bias (a), reliability (b), ROC (c), and CRPSS (d) metrics calculated from the reforecasts.

936

937 

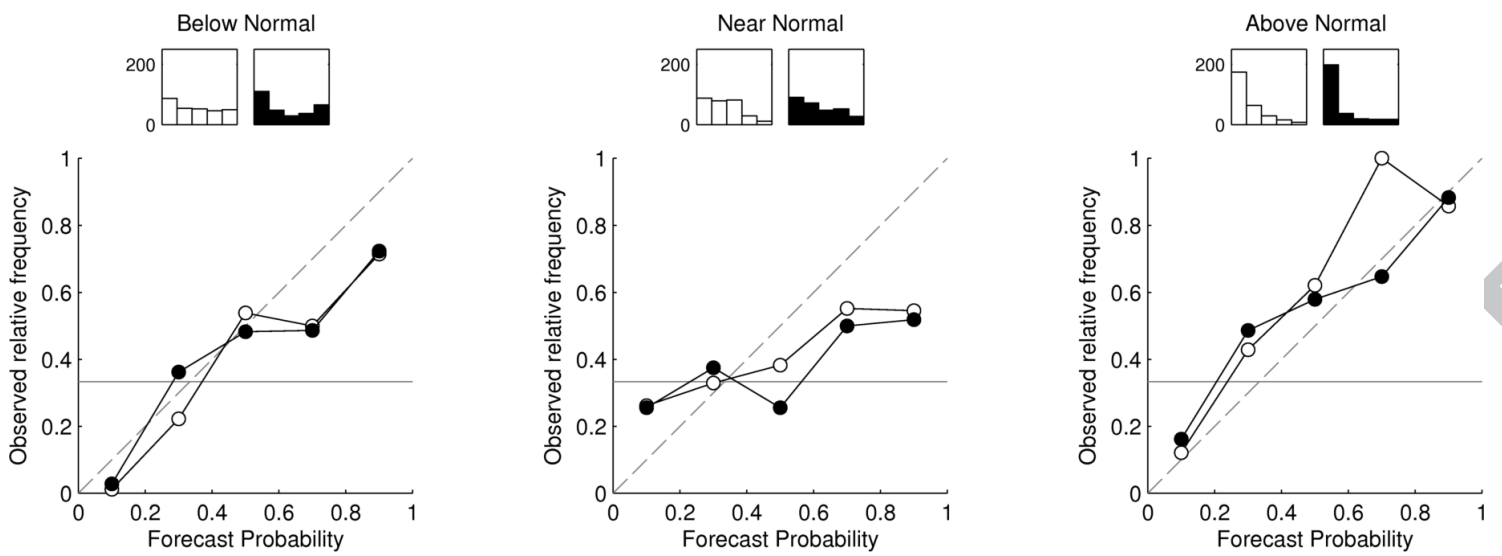

938

- GloSea O Climatology - - Perfect reliability —- Base rate

939 Figure 9: Reliability diagrams for the quickly responding catchments. The histograms denote the sample sizes

940 for each point on the reliability curves.

941

942

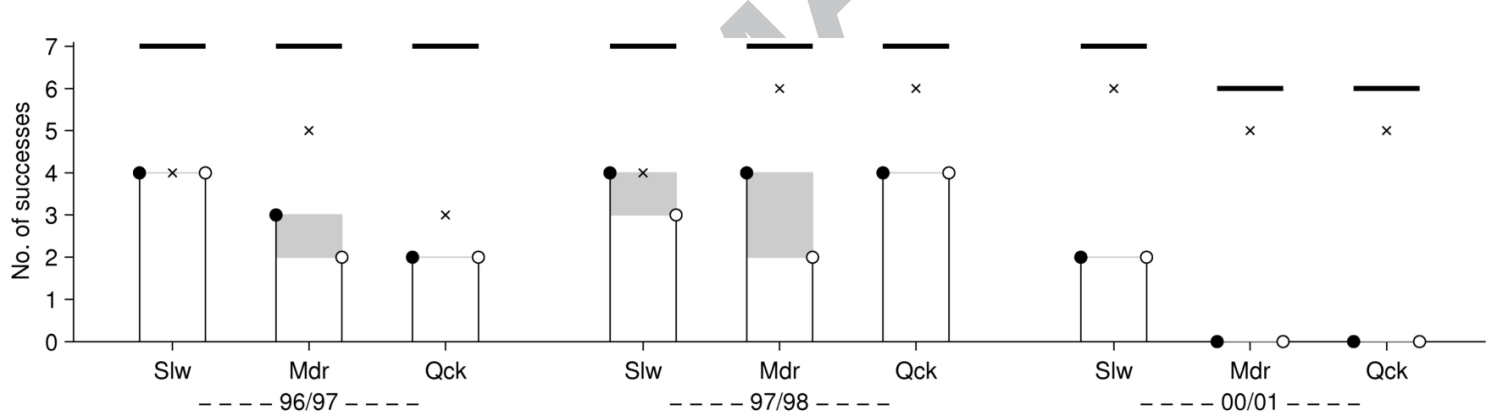

943

- GloSea $\times$ Observed $\bigcirc$ Climatology $\quad$ GloSea-climatology range

944 Figure 10: Number of successful forecasts for three events. The solid black lines indicate the total number of

945 catchments with available observation data.

946 


\section{ACCEPTED MANUSCRIPT}
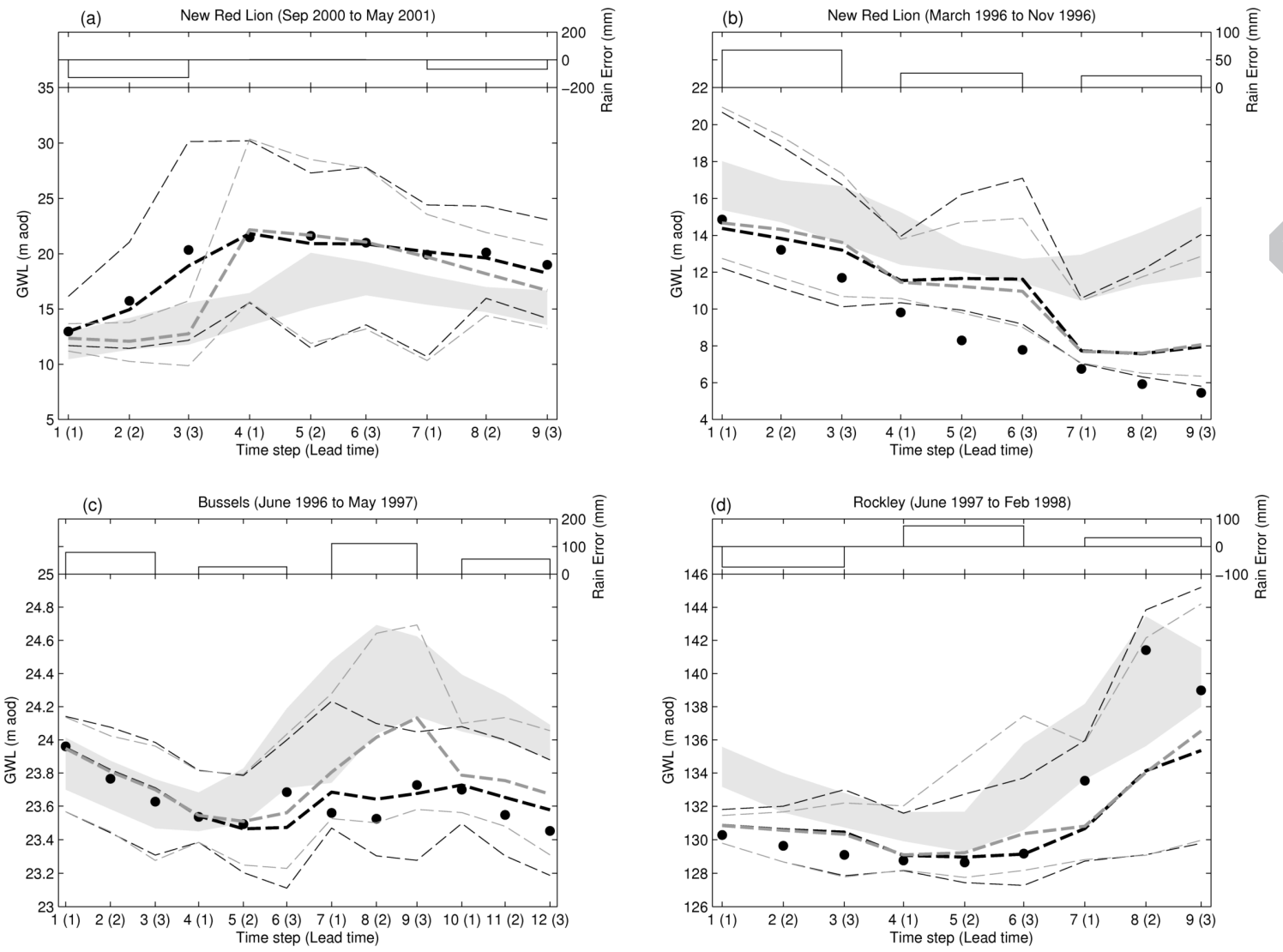

947

- Observations - - Ens. mean (GloSea rain) -

Unc. bounds (GloSea rain) -- Ens. mean (Obs. rain) --- Unc. bounds (Obs. rain)

Near normal levels

Figure 11: Comparison of the reforecasts using downscaled GloSea and observed rainfall inputs for different 
Table 1: List of AquiMod model parameters and calibration ranges.

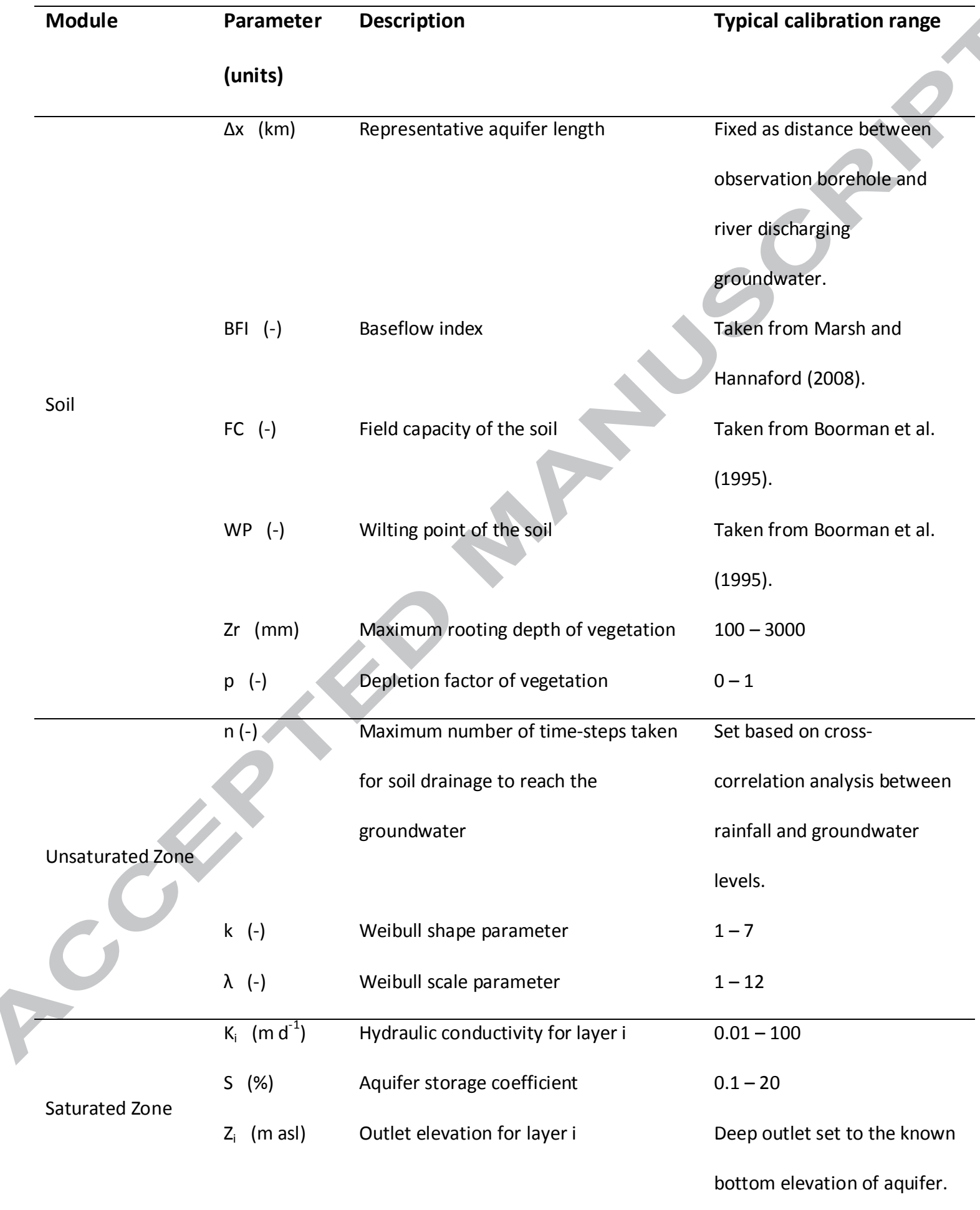


Remaining outlet elevations

set after preliminary

calibration runs.

960

961

962

963

964

965

966

967

968

969

970

971

972

973

974

975

976

977

978

979

980 
981 Table 2: List of 21 observation boreholes with the number of behavioural models (n), the efficiency of the most

982 efficient model (NSE $\max$ ) and the containment ratio using the GLUE 95\% confidence bounds (CR).

\begin{tabular}{|c|c|c|c|c|}
\hline Observation borehole & Aquifer & $\mathbf{n}$ & $\mathrm{NSE}_{\max }$ & CR \\
\hline Ashton Farm & Chalk & 2155 & 0.89 & 94.4 \\
\hline Aylesby & Chalk & 2470 & 0.82 & 96.9 \\
\hline Chilgrove House & Chalk & 2125 & 0.91 & 97.8 \\
\hline Clanville Lodge & Chalk & 2025 & 0.84 & 89.0 \\
\hline Dalton Holme & Chalk & 2000 & 0.81 & 82.6 \\
\hline Grimes Graves & Chalk & 1960 & 0.86 & 88.9 \\
\hline Little Bucket Farm & Chalk & 2305 & 0.90 & 85.7 \\
\hline Rockley & Chalk & 1835 & 0.88 & 94.1 \\
\hline Stonor Park & Chalk & 2430 & 0.78 & 65.3 \\
\hline Therfield Rectory & Chalk & 1915 & 0.71 & 68.9 \\
\hline Washpit Farm & Chalk & 1910 & 0.91 & 96.3 \\
\hline Well House Inn & $\mathrm{ch}$ & 1850 & 0.73 & 68.1 \\
\hline West Dean & Cha & 2210 & 0.83 & 92.2 \\
\hline West Woodyat & Chalk & 1780 & 0.86 & 84.8 \\
\hline New Rec & Jurassic Limestone & 2155 & 0.74 & 77.0 \\
\hline Lower Barn Cott & Lower Greensand & 2120 & 0.81 & 79.5 \\
\hline Swan House & Magnesian Limestone & 1960 & 0.86 & 89.6 \\
\hline Bussels & Permo-Triassic Sandstone & 2090 & 0.94 & 97.5 \\
\hline Furness Abbey & Permo-Triassic Sandstone & 2055 & 0.75 & 72.7 \\
\hline Heathlanes & Permo-Triassic Sandstone & 2095 & 0.87 & 87.9 \\
\hline Skirwith & Permo-Triassic Sandstone & 2390 & 0.83 & 87.6 \\
\hline
\end{tabular}


983

984

985

986

987 Highlights

988

- We forecast ground water levels 3 months into the future for 21 boreholes in the UK.

989

- We use GloSea5 seasonal rainfall forecasts to drive a conceptual groundwater

990 model.

991

- The forecasts consistently show more skill than a persistence forecasting approach.

992 - The forecasts are not able to capture extreme ground water level events.

993 - Sensitivity to (skill derived from) rainfall forecasts is highly site specific.

994 